Review Article

\title{
Efficient Single Image Dehazing Model Using Metaheuristics-Based Brightness Channel Prior
}

\author{
Vinay Kehar $\mathbb{D}^{1},{ }^{1}$ Vinay Chopra $\mathbb{D}^{2},{ }^{2}$ Bhupesh Kumar Singh $\mathbb{D}^{3},{ }^{3}$ and Shailendra Tiwari $\mathbb{D}^{4}$ \\ ${ }^{1}$ Department of Computer Science and Engineering, I. K. Gujral Punjab Technical University, Kapurthala, Punjab, India \\ ${ }^{2}$ Department of Computer Science and Engineering, DAV Institute of Engineering and Technology, Jalandhar 144008, India \\ ${ }^{3}$ Faculty of Computing and Software Engineering, Arba Minch University, Arba Minch, Ethiopia \\ ${ }^{4}$ Department of Computer Science \& Engineering, Thapar Institute of Engineering and Technology, Patiala, India
}

Correspondence should be addressed to Bhupesh Kumar Singh; dr.bhupeshkumarsingh@gmail.com

Received 23 February 2021; Revised 28 March 2021; Accepted 23 April 2021; Published 8 May 2021

Academic Editor: Hassène Gritli

Copyright ( $\odot 2021$ Vinay Kehar et al. This is an open access article distributed under the Creative Commons Attribution License, which permits unrestricted use, distribution, and reproduction in any medium, provided the original work is properly cited.

\begin{abstract}
Haze degrades the spatial and spectral information of outdoor images. It may reduce the performance of the existing imaging models. Therefore, various visibility restoration models approaches have been designed to restore haze from still images. But restoring the haze is an open area of research. Although the existing approaches perform significantly better, they are not so effective against a large haze gradient. Also, the effect of hyperparameters tuning issue is also ignored. Therefore, a brightness channel prior (BCP) based dehazing model is proposed. The gradient filter is utilized to improve the transmission map computed using the gradient filter. Nondominated Sorting Genetic Algorithm is also used to optimize the initial parameters of the BCP approach. The comparative analysis shows that BCP performs effectively across a wide range of haze degradation levels without causing any visible artifacts.
\end{abstract}

\section{Introduction}

Images captured in poor environmental conditions, such as haze, fog, and smoggy, suffer from poor visibility issues $[1,2]$. The haze attenuates the scene radiance with correspondence to an object's distance from the camera $[3,4]$. The haze imaging model is defined as a linear per-pixel consolidation of an original scene radiance and an airlight $[5,6]$.

Various multiple-images based haze restoration approaches have been implemented [7]. These approaches require physical characteristics of input images in prior [8-10]. But in real life, no physical attributes of input images are available in prior $[11,12]$.

Many techniques have been designed in the literature to remove haze from still images. Oakley and Satherley [13] designed a physical model to restore weather degraded images. The depth map and atmospheric veil were estimated to remove the visibility degradation from weather degraded images. It discovers the law of weather degraded image formation by considering the visual manifestations under various environmental circumstances [14]. Due to the extensive computational complexity of the physical model, He et al. [15] implemented a novel channel prior, that is, dark channel prior (DCP). It assumes that, for an image taken in a sunny environment, the intensity of at least one-color channel approaches toward zero. However, DCP suffers from a number of problems such as sky-region, halo, and gradient-reversal artifacts, color, edges, and texture distortion issues [16]. Recently, researchers have proposed various channel priors to handle the issues associated with standard DCP such as boosting dark channel [17], bounded optimization-based dark channel prior [18], gradient channel prior [19], adaptive bichannel priors [20], sparse dark channel prior [21], and dark channel prior guided variational framework [22]. However, the existing methods perform poorly especially when images contain a large haze gradient. Most of the existing methods suffer from texture distortion issues [23, 24].

Guo et al. [25] proposed a fusion model to restore the foggy images. It has shown significantly better edge and color preservation. Yoon [26] implemented a variational minimization based haze restoration model. However, $[25,26]$ are computationally expensive in nature [27]. 
The primary goal of this research work is to overcome the dehazing artifacts and to preserve significant information of restored images. Brightness channel prior (BCP) is used to obtain the physical attributes of hazy images. The gradient filter is used to refine the transmission map computed using the gradient filter. To optimize the initial parameters of BCP, NSGA is utilized. The comparative analysis is also drawn to evaluate the performance of NSGA based BCP model.

The rest of the paper is organized as follows: Section 2 discusses the related work. The proposed model is presented in Section 3. The comparative analysis is presented in Section 4. Section 5 concludes the proposed haze restoration model.

\section{Related Works}

Luan et al. [28] proposed a restoration model by using the regression model. Support vector regression is used for regression model learning. Jiang et al. [20] proposed an adaptive bichannel prior to superpixels for removing haze from the single image. The superpixels are used as local regions to estimate the atmospheric light and transmission map by combining bright and dark channel priors. Liu et al. [29] utilized a multiscale correlated wavelet approach for restoring the weather degraded images. In multiscale wavelet decomposition, it has been found that haze mainly presents in the low-frequency spectrum. To remove the haze effect, an open dark channel model (ODCM) is used.

Nair and Sankaran [14] implemented a haze removal approach using a dark channel prior and surround filter. The computational complexity of the approach is due to simple convolution. The surround filter minimizes the memory requirements and enhances the speed of transmission estimation. Wu et al. [30] proposed a restoration model for UAV-based railway images using a densely pyramidal residual network (DPRnet). The loss function is used to preserve the structural information significantly. Shu et al. [31] used a multichannel total variation (MTV) regularizer to restore the hazy images. The alternating direction approach of multipliers is utilized for a nonsmooth optimization problem.

Hodges et al. [32] developed a deep learning-based restoration model (DLR) of weather degraded images. In this, a deep learning model is utilized to train the samples using unmatched images. Zhang et al. [33] solved the problem of bright distortion due to DCP. To eliminate the bright distortion, four parameters, such as mean square error (MSE), mean gradient, program running time, and peak signal-to-noise ratio (PSNR), are evaluated optimally. The logarithmic enhancement approach is used as an optimal approach.

Emberton et al. [34] used haze region segmentation (SRS) to remove haze from images. To address the problem of spectral distortion, a semantic white balancing approach is applied. Guo et al. [35] utilized the deep convolutional network (DCN) to remove the haze from images. Five maps are derived from the original hazy image. The saliency map and exposure map are used to focus on near-region scenes. The gamma correction map and white balance map are applied to gain the components of the intensity and latent color of the scene. The global image contrast is enhanced by using the haze veil map.

Alajarmeh et al. [36] proposed an image restoration model based on contrast time airlight and liner transmission (CLT). Two approaches are used such as airlight by image integrals to estimate the airlight value and bounded transmission to estimate the linear transmission maps. Gao et al. [37] proposed a dual-fusion approach (DFT) to restore the hazy images. The segmentation approach is used to divided the regions such as the sky and nonsky. A multiregion fusion approach is used to optimally evaluate the transmission map.

From the existing review, it has been observed that the hyperparameters tuning of the dehazing models can achieve better results [38, 39]. In this paper, we have considered various metaheuristic techniques such as Multiobjective Harris Hawks Optimizer (MOHO) [40], Multiobjective Extremal Optimization (MOEO) [41], Multiobjective Particle Swarm Optimization (MOPSO) [42], Multiobjective Grey Wolf Optimizer (MOGWO) [43], Multiobjective Whale Optimization (MOWO) [44], and Nondominated Sorting Genetic Algorithm (NSGA)-III [45]. All these approaches are utilized in this paper to tune the initial parameters of GCP by designing a multiobjective fitness function.

\section{Proposed Brightness Channel Prior-Based Dehazing Approach}

This section discusses the designed dehazing model. Figure 1 demonstrates the overall flow of the designed dehazing model.

3.1. Depth Map Estimation. In the first step, BCP is defined to approximate the depth map of a hazy image $\left(H_{i}\right)$ as

$$
I^{d}(p, q)=\delta_{y \in \Psi(p, q)}\left(\delta_{c \in(r, g, b)}\left(I_{s}^{c}(l)\right)\right),
$$

where $\Psi(p, q)$ defines local patch. $\delta$ shows BCP. $I_{m}^{c}$ defines color channels of $H_{i}$.

3.2. Atmospheric Veil. Atmospheric veil $(\gamma)$ is then estimated as [15]

$$
\gamma(p, q)=H_{i}\left(\max _{c}\left(I_{m}^{c}\right)\right)
$$

3.3. Transmission Map. Transmission map $(\tilde{t})$ is then evaluated by using the computed BCP as

$$
\tilde{t}(p, q)=1-\min _{y \in \Psi(p, q)}\left(\min _{c} \frac{I_{m}^{c}(y)}{A_{l}^{c}}\right) .
$$

3.4. Coarse Atmospheric Veil. The coarse atmospheric veil $\left(A_{v}(p, q)\right)$ is then computed as [15] 


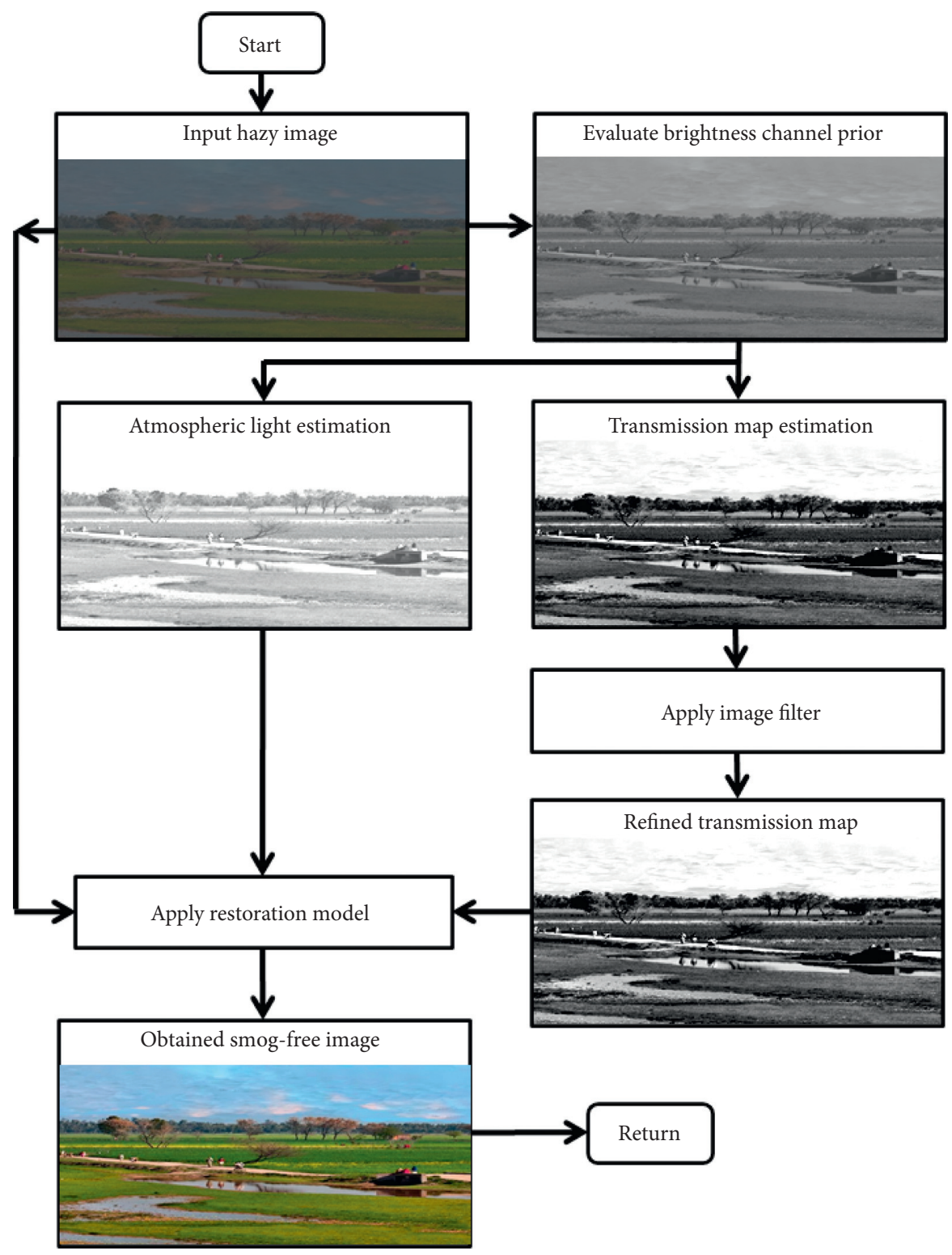

FIGURE 1: Flowchart of brightness channel prior based dehazing approach.

$$
A_{v}(p, q)=\beta \min _{y \in \Psi(p, q)}\left(\min _{c} \frac{I_{m}^{c}(y)}{A_{l}^{c}}\right)
$$

In this paper, a gradient filter is used to improve $t$ as

$$
\widetilde{t}(p, q)=\sigma(p, q)-J_{O}^{t f}(|t-\sigma(p, q)|)
$$

where $\sigma(p, q)$ defines the standard deviation.

3.5. Restoration Model. In the last step of BCP, a haze-free image $\left(C_{m}\right)$ can be evaluated as

$$
C_{m}(p, q)=\frac{H_{i}(p, q)-\gamma}{\max \left(\tilde{t}(p, q), t_{l}\right)}+\gamma
$$

3.6. Optimization of Initial Parameters of BCP. To optimize the initial parameters of BCP, NSGA [46] is used. Flowchart of NSGA based BCP is depicted in Figure 2.

NSGA-III [45] has been extensively utilized to solve many computationally complex problems. NSGA-III is preferred over the existing multiobjective optimization approaches as it has good convergence speed and it does not suffer from premature convergence issues [47-49]. It utilizes nondominated sorting to sort the nondominated solutions. Table 1 shows the nomenclature of NSGA-III.

Algorithm 1 demonstrates the initial population of NSGA-III based BCP. Initially, a random population is computed. The obtained solutions are then encoded to the range of hyperparameters of BCP. Algorithm 2 demonstrates the working of the proposed NSGA-III based BCP. New visible edges, saturated pixels, and new edge gradients are 


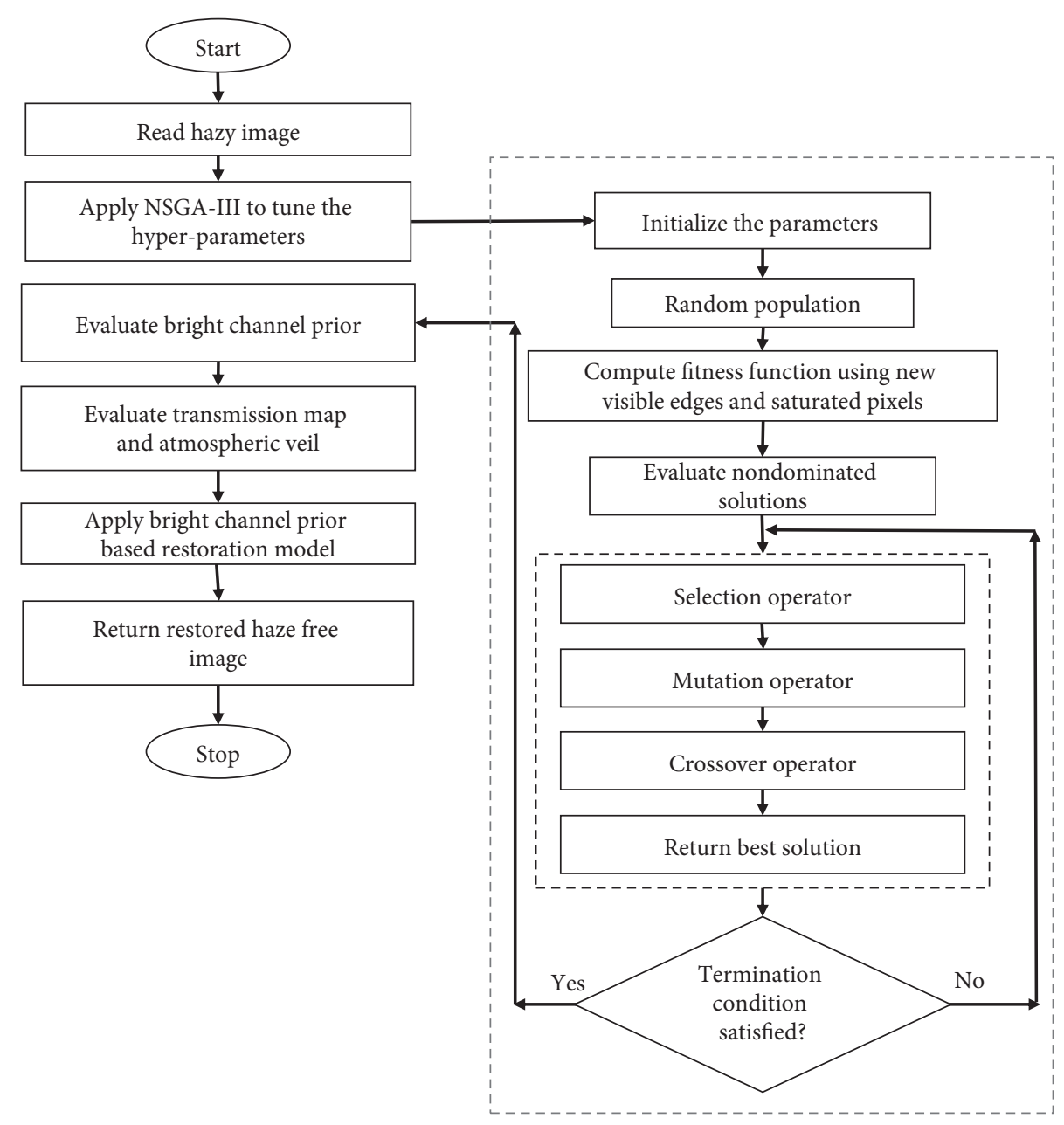

FIGURE 2: NSGA based parameters optimization of BCP model.

TABLE 1: Nomenclature of NSGA-III

\begin{tabular}{lc}
\hline Symbol & Meaning \\
\hline $\mathscr{K}$ & Random set of solutions \\
$\mathscr{R}$ & Group of $\tau^{\prime}, \kappa^{\prime \prime}$ \\
$\alpha$ & Random variable $\in \in[0,1]$ \\
$\tau$ & Permutation vector \\
$\zeta$ & Encoding \\
$\mathscr{E}_{t}$ & Elite population \\
$\tau$ & Optimal BCP \\
$\kappa$ & Optimal parameters \\
$\kappa$ & Binary decision vector \\
\hline
\end{tabular}

used as a fitness function. Dominated and nondominated solutions are then computed. Crossover and mutation operators are then used to compute the child solutions. Nondominated sorting is then implemented by using the dominance relation (). Finally, when termination criteria are achieved, then the optimal initial parameter of BCP is returned.

$\zeta(\tau, \kappa)$ decompose random individual $(\tau, \kappa)$ to a hyperparameters of BCP.

\section{Performance Analysis}

The performance of the NSGA-BCP dehazing model is evaluated on $i 7$ processor with $2.66 \mathrm{GHz}$ and $16 \mathrm{~GB}$ RAM. MATLAB $2019 a$ is used to perform the experiments. The patch size is selected as $3 \times 3$ pixels. Seven well-known dehazing approaches are used for comparative analyses. These approaches are DCP [50], CTT [51], CNN [52], WT [53], FVID [54], $L_{1}$ norm [55], and TGV [56]. Fifteen benchmark synthetic and real-life hazy images are considered for experimental analysis.

4.1. Visual Analyses of Proposed Dehazing Model. Figures 3-5 show the visual analysis. Restored images obtained from DCP [15] and CTT [51] show distortion of edge and texture information. Also, DCP [15] and CTT [51] suffer from the sky-region problem. CNN [52] and TGV [56] provide better results, but still some texture distortion is there. WT [53], $L_{1}$ norm [55], and FVID [54] suffer from some halo and gradient-reversal artifacts. The NSGA-BCP based restored images preserve the edge, texture, and color details efficiently. 

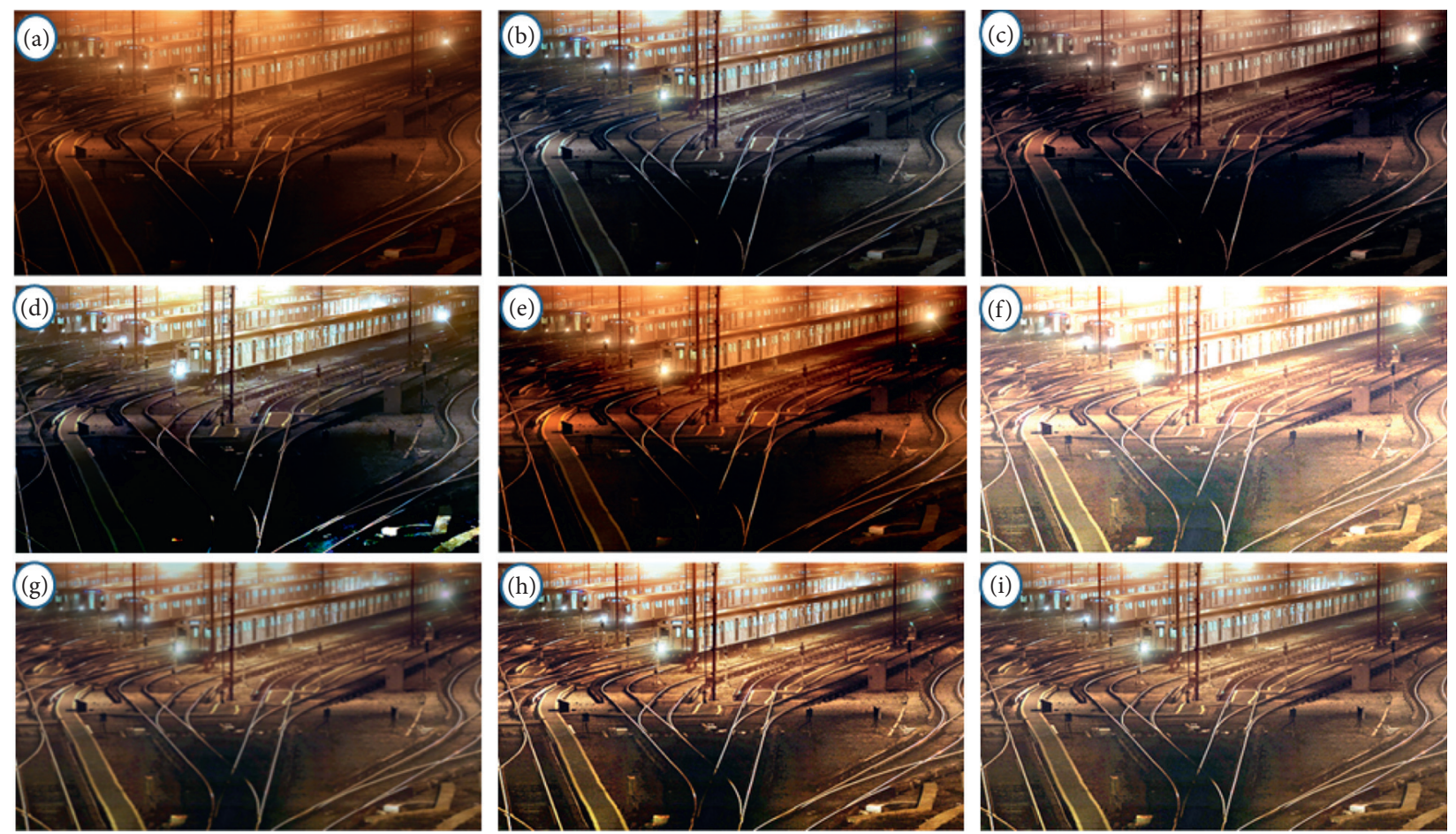

Figure 3: Results of dehazing models: (a) input image, (b) DCP [50], (c) CNN [52], (d) CTT [51], (e) TGV [56], (f) WT [53], (g) $L_{1}$ norm [55], (h) FVID [54], and (i) proposed BCP model.
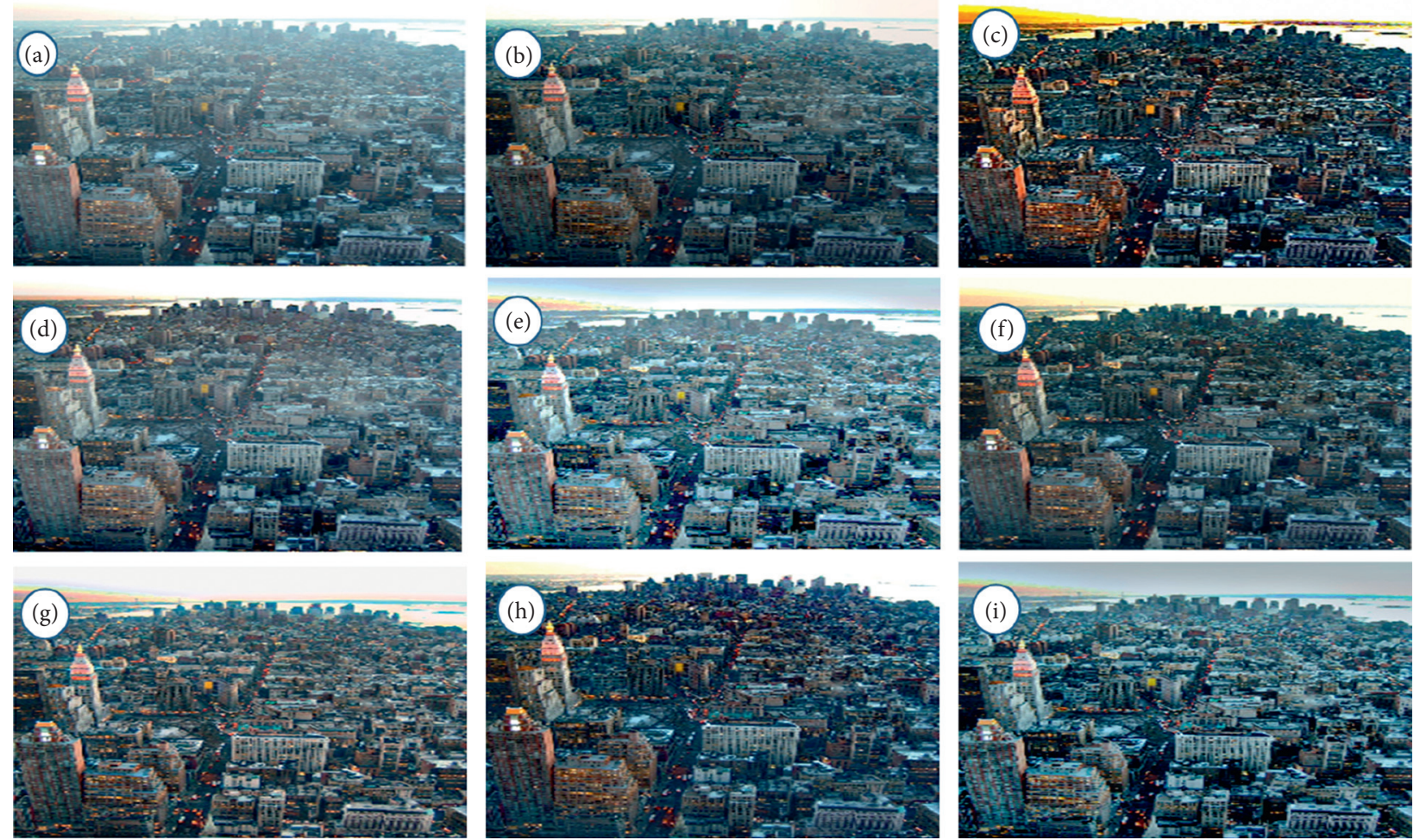

Figure 4: Results of dehazing models: (a) input image, (b) DCP [50], (c) CNN [52], (d) CTT [51], (e) TGV [56], (f) WT [53], (g) $L_{1}$ norm [55], (h) FVID [54], and (i) proposed BCP dehazing model.

4.2. Quantitative Analyses of Proposed Dehazing Model. The proposed model is compared with the competitive models by considering various performance metrics like percentage of saturated pixels $\left(S_{p}\right)$, haze gradient, contrast gain (CG), visible edges, execution time (ET), structural similarity index metric, and peak signal-to-noise ratio. Bold 

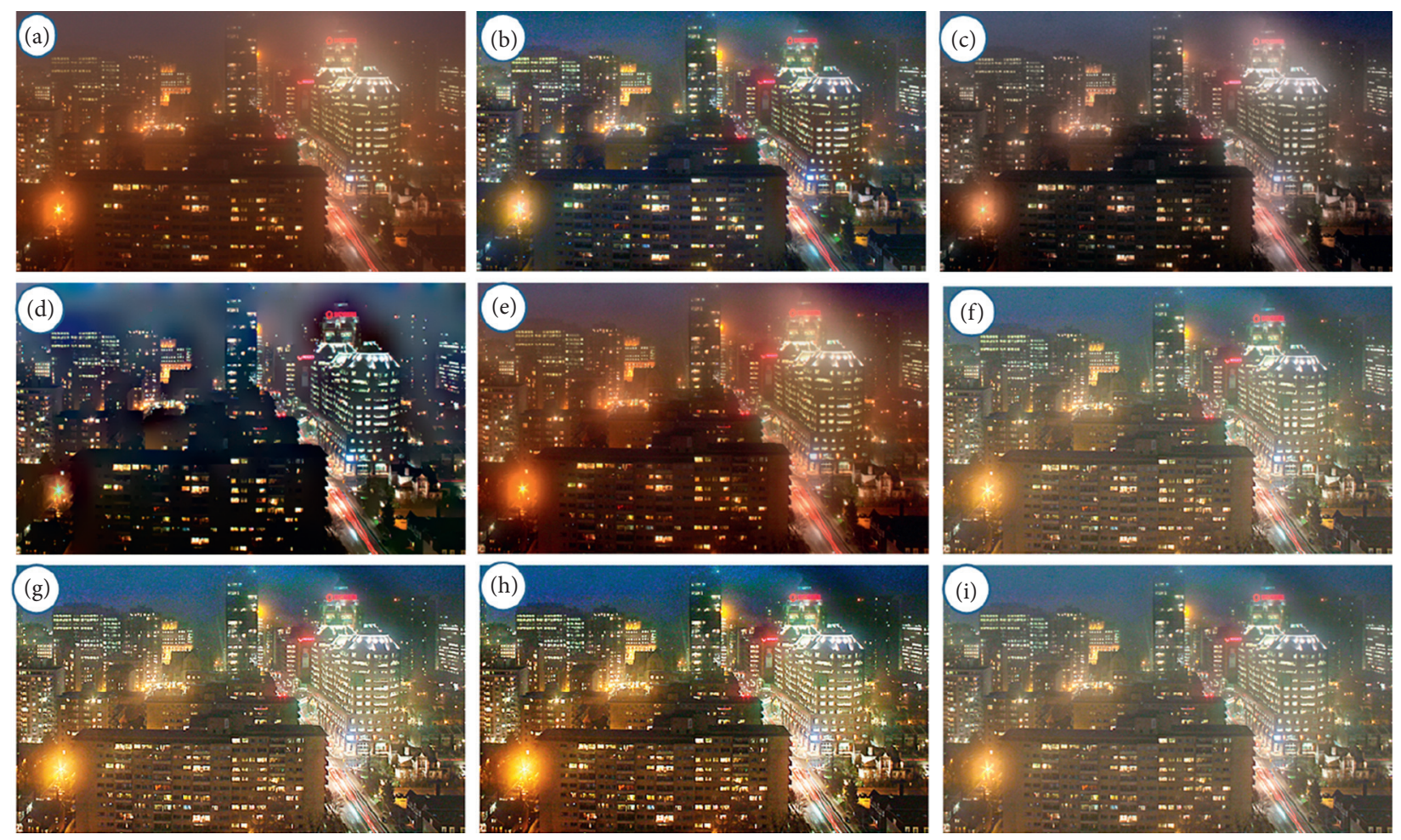

Figure 5: Results of dehazing models: (a) input image, (b) DCP [50], (c) CNN [52], (d) CTT [51], (e) TGV [56], (f) WT [53], (g) $L_{1}$ norm [55], (h) FVID [54], and (i) proposed BCP model.

values in the table represent the high performance of the given model.

Table 2 demonstrates CG analysis. It is found that the proposed NSGA-BCP based dehazing model has significant CG values compared to the competitive dehazing approaches. Overall CG analysis shows that the proposed model achieves an average of 1.9745 CG as compared to the maximum average CG, that is, 1.8932, obtained using the existing dehazing models.

Table 3 reveals that the proposed NSGA-BCP based dehazing model has minimum $S_{p}$ values compared to the competitive dehazing models. Overall $S_{P}$ analysis shows that the proposed model achieves an average of $0.0226 S_{p}$ which is significantly lesser as compared to the minimum average $S_{p}$, that is, 0.0463 obtained using the existing dehazing models.

Tables 4 and 5 demonstrate that the proposed NSGABCP based dehazing model has achieved better $e$ and $\bar{r}$ values as compared to the existing approaches. Overall $e$ analysis shows that the proposed model achieves an average of $2.9426 e$ which is significantly better as compared to the maximum average $e$, that is, 2.7367 , obtained using the existing dehazing models. Overall $\bar{r}$ analysis shows that the proposed model achieves an average of $3.0173 \bar{r}$ which is significantly better as compared to the maximum average $\bar{r}$, that is, 2.7363, obtained using the existing dehazing models.

Table 6 demonstrates execution time (in seconds) analysis. It is observed that the proposed NSGA-BCP based dehazing model is computationally faster than the existing approaches. Overall execution time analysis shows that the proposed model achieves an average of 1.0324 execution time which is significantly minimum as compared to the minimum average execution time, that is, 1.3853 , obtained using the existing dehazing models.

Table 7 demonstrates haze gradient analyses. It is observed that the NSGA-BCP based dehazing model achieves lesser haze gradient values as compared to the existing approaches. Overall haze gradient analysis shows that the proposed model achieves an average of 1.7483 haze gradient which is significantly minimum as compared to the minimum average haze gradient, that is, 1.9485 , obtained using the existing dehazing models.

Table 8 shows PSNR analyses of the proposed and the competitive dehazing models. It is found that the proposed NSGA-BCP based dehazing model has significant PSNR values than the competitive dehazing approaches. Overall PSNR analysis shows that the proposed model achieves an average of 27.9136 PSNR which is significantly lesser as compared to the maximum average PSNR, that is, 23.2943, obtained using the existing dehazing models.

Table 9 shows SSIM analyses of the proposed and the competitive dehazing models. It is found that the proposed NSGA-BCP based dehazing model has significant SSIM values than the competitive dehazing approaches. Overall SSIM analysis shows that the proposed model achieves an average of 0.8932 SSIM which is significantly lesser as compared to the maximum average SSIM, that is, 0.8395, obtained using the existing dehazing models.

From Tables 2-10, it has been found that the NICP outperforms the competitive dehazing models in terms of 


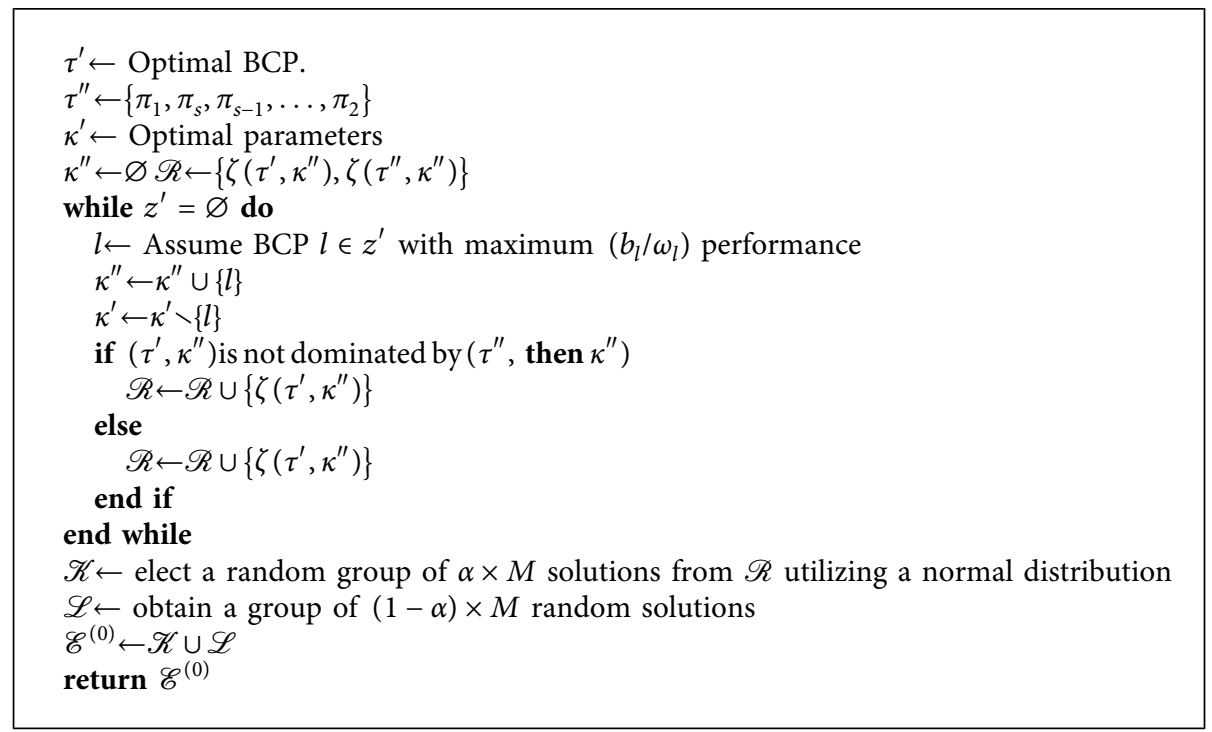

Algorithm 1: Obtain initial population.

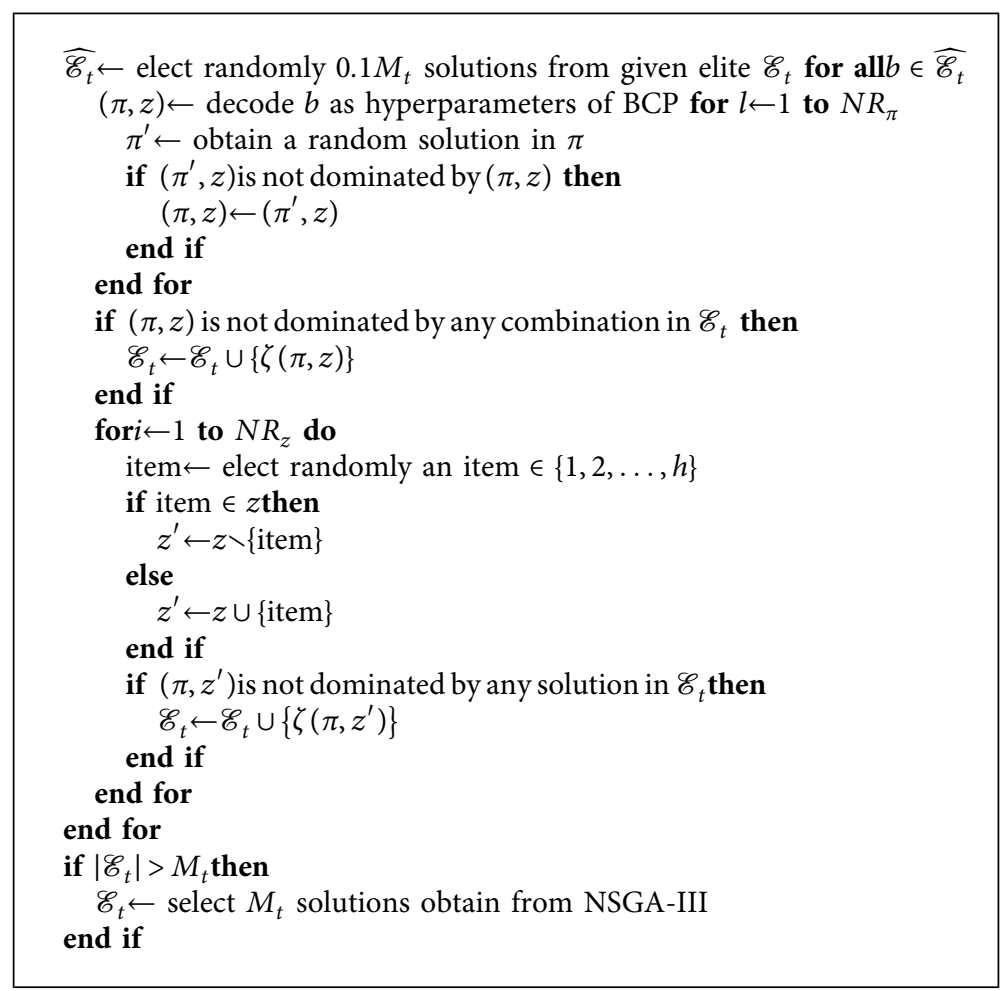

Algorithm 2: Proposed NSGA-III based BCP.

contrast gain (CG), new visible edges (NVE), average gradient (AG), peak signal-to-noise ratio (PSNR), and structural similarity index metric (SSIM) by $1.2883 \%, 1.5392 \%$, $0.8271 \%, 0.8928 \%$, and $1.2813 \%$, respectively. Compared to the competitive models, NICP also minimizes the haze gradient, saturated pixels, and execution time by $0.8282 \%$, $0.7291 \%$, and $1.1428 \%$, respectively.
Besides this, we have also compared the performance of the proposed model with the different optimization approaches such as Multiobjective Harris Hawks Optimizer (MOHO) [40], Multiobjective Extremal Optimization (MOEO) [41], Multiobjective Particle Swarm Optimization (MOPSO) [42], Multiobjective Grey Wolf Optimizer (MOGWO) [43], and Multiobjective Whale Optimization 
TABLE 2: Contrast gain analyses of the proposed dehazing model.

\begin{tabular}{|c|c|c|c|c|c|c|c|c|}
\hline Img. & DCP & FVID & WT & TGV & CTT & $L_{1}$ & CNN & NSGA-BCP \\
\hline $\mathrm{IM}_{1}$ & 1.8745 & 1.8432 & 1.7745 & 1.8564 & 1.8745 & 1.8654 & 1.8856 & 1.9631 \\
\hline $\mathrm{IM}_{2}$ & 1.8756 & 1.8245 & 1.8754 & 1.8923 & 1.8156 & 1.7923 & 1.8125 & 1.9149 \\
\hline $\mathrm{IM}_{3}$ & 1.8749 & 1.7932 & 1.8830 & 1.8623 & 1.8923 & 1.7158 & 1.8856 & 1.9198 \\
\hline $\mathrm{IM}_{4}$ & 1.8752 & 1.8584 & 1.8523 & 1.8864 & 1.8625 & 1.8523 & 1.8456 & 1.9158 \\
\hline $\mathrm{IM}_{5}$ & 1.8285 & 1.8546 & 1.8524 & 1.8423 & 1.8518 & 1.8523 & 1.8523 & 1.9125 \\
\hline $\mathrm{IM}_{6}$ & 1.8823 & 1.8569 & 1.8569 & 1.8654 & 1.8853 & 1.8597 & 1.8829 & 1.9065 \\
\hline $\mathrm{IM}_{7}$ & 1.7926 & 1.7957 & 1.7859 & 1.7745 & 1.7852 & 1.8746 & 1.8489 & 1.9875 \\
\hline $\mathrm{IM}_{8}$ & 1.8531 & 1.8547 & 1.8687 & 1.8741 & 1.8568 & 1.8741 & 1.8847 & 1.9746 \\
\hline $\mathrm{IM}_{9}$ & 1.8741 & 1.8654 & 1.8852 & 1.8159 & 1.8547 & 1.8624 & 1.8357 & 1.9025 \\
\hline $\mathrm{IM}_{10}$ & 1.7788 & 1.8759 & 1.8748 & 1.8475 & 1.8897 & 1.8547 & 1.8749 & 1.9568 \\
\hline $\mathrm{IM}_{11}$ & 1.8956 & 1.8983 & 1.8642 & 1.8554 & 1.8759 & 1.7982 & 1.8369 & 1.9546 \\
\hline $\mathrm{IM}_{12}$ & 1.8412 & 1.8547 & 1.8963 & 1.8858 & 1.8859 & 1.8654 & 1.8521 & 1.9214 \\
\hline $\mathrm{IM}_{13}$ & 1.8624 & 1.8512 & 1.8456 & 1.8258 & 1.8258 & 1.8245 & 1.8014 & 1.9078 \\
\hline $\mathrm{IM}_{14}$ & 1.8214 & 1.8852 & 1.8369 & 1.8147 & 1.8325 & 1.8512 & 1.8521 & 1.9756 \\
\hline $\mathrm{IM}_{15}$ & 1.8412 & 1.8654 & 1.8647 & 1.8025 & 1.8745 & 1.8742 & 1.8854 & 1.9789 \\
\hline
\end{tabular}

TABle 3: Saturated pixels ( $S_{p}$ analyses of the proposed dehazing model).

\begin{tabular}{lcccccccc}
\hline Img. & DCP & FVID & WT & TGV & CTT & $L_{1}$ & CNN & NSGA-BCP \\
\hline $\mathrm{IM}_{1}$ & 0.0568 & 0.0745 & 0.0456 & 0.0235 & 0.0547 & 0.0745 & 0.0478 \\
$\mathrm{IM}_{2}$ & 0.0958 & 0.0456 & 0.0745 & 0.0766 & 0.0456 & 0.0258 & 0.0178 & 0.0188 \\
$\mathrm{IM}_{3}$ & 0.0741 & 0.1258 & 0.2456 & 0.1854 & 0.2324 & 0.1478 & 0.1147 & 0.0115 \\
$\mathrm{IM}_{4}$ & 0.0514 & 0.1478 & 0.2149 & 0.2147 & 0.2759 & 0.2148 & 0.1789 & 0.0231 \\
$\mathrm{IM}_{5}$ & 0.0148 & 0.1148 & 0.2148 & 0.1258 & 0.2185 & 0.1446 & 0.1258 \\
$\mathrm{IM}_{6}$ & 0.0459 & 0.1748 & 0.2479 & 0.2654 & 0.1412 & 0.2128 & 0.1741 \\
$\mathrm{IM}_{7}$ & 0.0147 & 0.2459 & 0.2147 & 0.2852 & 0.1743 & 0.2547 & 0.1423 & 0.0354 \\
$\mathrm{IM}_{8}$ & 0.0413 & 0.2569 & 0.1312 & 0.1789 & 0.2147 & 0.2321 & 0.1426 & 0.0521 \\
$\mathrm{IM}_{9}$ & 0.0874 & 0.2652 & 0.2741 & 0.1123 & 0.1258 & 0.2654 & 0.2742 \\
$\mathrm{IM}_{10}$ & 0.0742 & 0.1589 & 0.2742 & 0.2842 & 0.2742 & 0.2459 & 0.1424 & 0.0214 \\
$\mathrm{IM}_{11}$ & 0.0759 & 0.1258 & 0.1512 & 0.1742 & 0.1358 & 0.2847 & 0.1426 \\
$\mathrm{IM}_{12}$ & 0.0456 & 0.2348 & 0.2428 & 0.1759 & 0.1783 & 0.1524 & 0.1478 & 0.0561 \\
$\mathrm{IM}_{13}$ & 0.0648 & 0.1621 & 0.1759 & 0.1247 & 0.1897 & 0.2358 & 0.2478 \\
$\mathrm{IM}_{14}$ & 0.0587 & 0.1248 & 0.1324 & 0.2348 & 0.1214 & 0.1856 & 0.2574 & 0.0248 \\
$\mathrm{IM}_{15}$ & 0.0654 & 0.2412 & 0.1324 & 0.1214 & 0.1225 & 0.1247 & 0.2415 & 0.0480 \\
\end{tabular}

TABLe 4: New visible edges analyses of the proposed dehazing model.

\begin{tabular}{|c|c|c|c|c|c|c|c|c|}
\hline Img. & DCP & FVID & WT & TGV & CTT & $L_{1}$ & $\mathrm{CNN}$ & NSGA-BCP \\
\hline $\mathrm{IM}_{1}$ & 2.0076 & 2.6048 & 1.7726 & 2.1789 & 2.5778 & 1.9857 & 1.9758 & 2.9301 \\
\hline $\mathrm{IM}_{2}$ & 2.5187 & 2.8321 & 2.6789 & 2.7412 & 2.6546 & 2.1456 & 2.2325 & 3.0547 \\
\hline $\mathrm{IM}_{3}$ & 2.0324 & 2.2456 & 2.5154 & 2.6254 & 2.1789 & 2.2325 & 2.7471 & 3.0526 \\
\hline $\mathrm{IM}_{4}$ & 2.7326 & 1.8654 & 2.2258 & 1.7452 & 1.9857 & 1.8325 & 2.2402 & 3.0289 \\
\hline $\mathrm{IM}_{5}$ & 2.3507 & 2.3845 & 2.3245 & 2.0452 & 1.9245 & 2.6325 & 1.7654 & 2.9875 \\
\hline $\mathrm{IM}_{6}$ & 2.5725 & 2.7524 & 2.6589 & 1.8856 & 2.5146 & 2.0708 & 2.2301 & 2.9841 \\
\hline $\mathrm{IM}_{7}$ & 2.7456 & 2.6772 & 2.5546 & 1.8852 & 2.1265 & 1.856 & 2.5758 & 2.9854 \\
\hline $\mathrm{IM}_{8}$ & 2.1759 & 2.3478 & 2.3452 & 2.6254 & 2.8125 & 2.0088 & 2.1852 & 2.9742 \\
\hline $\mathrm{IM}_{9}$ & 1.9824 & 1.9654 & 2.3189 & 1.9248 & 2.3741 & 2.2544 & 2.1245 & 2.9547 \\
\hline $\mathrm{IM}_{10}$ & 2.3256 & 2.2145 & 2.2147 & 2.2485 & 1.9874 & 1.9856 & 2.0221 & 2.7456 \\
\hline $\mathrm{IM}_{11}$ & 2.5156 & 2.4985 & 1.8567 & 2.7998 & 2.8857 & 1.8742 & 1.8987 & 3.2148 \\
\hline $\mathrm{IM}_{12}$ & 2.4987 & 2.1952 & 2.5231 & 2.0324 & 2.1456 & 1.7987 & 2.1347 & 2.8534 \\
\hline $\mathrm{IM}_{13}$ & 2.5641 & 1.7852 & 1.9472 & 2.4652 & 2.3873 & 2.7814 & 2.6932 & 2.9856 \\
\hline $\mathrm{IM}_{14}$ & 2.2632 & 2.5214 & 2.1369 & 1.7963 & 2.5214 & 2.2266 & 2.6742 & 2.9872 \\
\hline $\mathrm{IM}_{15}$ & 2.4321 & 2.7421 & 2.7981 & 2.0325 & 2.2354 & 2.7561 & 2.5321 & 3.1456 \\
\hline
\end{tabular}


TABLE 5: Ratio of average gradient analyses of the proposed dehazing model.

\begin{tabular}{lcccccccc}
\hline Img. & DCP & FVID & WT & TGV & CTT & $L_{1}$ & CNN & NSGA-BCP \\
\hline $\mathrm{IM}_{1}$ & 2.1963 & 1.9856 & 2.1854 & 2.8624 & 2.6541 & 2.7896 & 2.8561 & 3.1856 \\
$\mathrm{IM}_{2}$ & 2.1452 & 2.1956 & 2.1852 & 2.1826 & 1.8452 & 2.0578 & 2.0436 \\
$\mathrm{IM}_{3}$ & 2.2756 & 2.4536 & 2.7852 & 2.5345 & 2.0175 & 2.6523 & 2.1102 \\
$\mathrm{IM}_{4}$ & 2.3245 & 2.3157 & 2.8563 & 2.6124 & 2.1475 & 2.7853 & 2.1632 & 3.1234 \\
$\mathrm{IM}_{5}$ & 2.5234 & 1.8242 & 2.8213 & 1.7894 & 2.5632 & 2.7753 & 2.6321 \\
$\mathrm{IM}_{6}$ & 1.9354 & 2.4536 & 2.4369 & 2.8874 & 1.9632 & 1.8789 & 1.9653 \\
$\mathrm{IM}_{7}$ & 2.8524 & 2.8456 & 2.4951 & 2.1654 & 2.3956 & 1.8632 & 2.5634 & 3.1456 \\
$\mathrm{IM}_{8}$ & 2.0085 & 2.3165 & 1.8632 & 2.4214 & 2.1532 & 1.8997 & 2.6547 & 3.1156 \\
$\mathrm{IM}_{9}$ & 2.2187 & 2.5845 & 2.1475 & 2.5817 & 2.2985 & 2.4123 & 2.7456 \\
$\mathrm{IM}_{10}$ & 2.1563 & 2.5521 & 2.7165 & 2.1254 & 2.5423 & 2.6756 & 2.4457 \\
$\mathrm{IM}_{11}$ & 2.4852 & 2.2881 & 2.5236 & 2.4756 & 2.1756 & 2.4681 & 2.1533 \\
$\mathrm{IM}_{12}$ & 2.6653 & 2.8745 & 2.2241 & 1.8854 & 1.7742 & 2.1587 & 2.1354 & 2.8567 \\
$\mathrm{IM}_{13}$ & 2.4325 & 2.4701 & 2.1324 & 2.0214 & 2.2147 & 2.6541 & 2.8521 \\
$\mathrm{IM}_{14}$ & 2.1856 & 1.8321 & 1.8572 & 2.7523 & 2.3753 & 2.7423 & 2.7145 \\
$\mathrm{IM}_{15}$ & 2.5321 & 2.2742 & 2.1253 & 1.8856 & 2.1258 & 2.7745 & 2.8230 & 3.1483 \\
\hline
\end{tabular}

TABLE 6: Execution time analyses of the proposed dehazing model.

\begin{tabular}{|c|c|c|c|c|c|c|c|c|}
\hline Img. & DCP & FVID & WT & TGV & CTT & $L_{1}$ & $\mathrm{CNN}$ & NSGA-BCP \\
\hline $\mathrm{IM}_{1}$ & 1.7532 & 1.3214 & 1.2941 & 1.2789 & 1.2143 & 1.1854 & 1.9874 & 1.0211 \\
\hline $\mathrm{IM}_{2}$ & 1.3214 & 1.3478 & 1.7852 & 1.0547 & 1.2847 & 1.1458 & 1.4412 & 1.0112 \\
\hline $\mathrm{IM}_{3}$ & 1.9765 & 1.8547 & 1.6475 & 1.5521 & 1.4874 & 1.6321 & 1.4365 & 1.2415 \\
\hline $\mathrm{IM}_{4}$ & 1.4842 & 1.3214 & 1.5641 & 1.8745 & 1.4123 & 1.0745 & 1.2777 & 1.0521 \\
\hline $\mathrm{IM}_{5}$ & 1.0742 & 1.4324 & 1.0547 & 1.7423 & 1.3547 & 1.3321 & 1.1324 & 1.0214 \\
\hline $\mathrm{IM}_{6}$ & 1.2567 & 1.2247 & 1.4658 & 1.3547 & 1.1187 & 1.3547 & 1.6571 & 1.1012 \\
\hline $\mathrm{IM}_{7}$ & 1.8423 & 1.3452 & 1.2310 & 1.5845 & 1.6621 & 1.6874 & 1.3276 & 1.1014 \\
\hline $\mathrm{IM}_{8}$ & 1.4741 & 1.2114 & 1.3214 & 1.1214 & 1.3841 & 1.3245 & 1.8623 & 1.0214 \\
\hline $\mathrm{IM}_{9}$ & 1.4321 & 1.3321 & 1.2214 & 1.4321 & 1.4632 & 1.4321 & 1.8287 & 1.1234 \\
\hline $\mathrm{IM}_{10}$ & 1.2456 & 1.5812 & 1.4632 & 1.2452 & 1.3214 & 1.7113 & 1.3214 & 1.1132 \\
\hline $\mathrm{IM}_{11}$ & 1.2547 & 1.2321 & 1.3756 & 1.4451 & 1.3475 & 1.1532 & 1.6321 & 1.0203 \\
\hline $\mathrm{IM}_{12}$ & 1.3214 & 1.5147 & 1.2147 & 1.1847 & 1.2214 & 1.2147 & 1.7145 & 1.1014 \\
\hline $\mathrm{IM}_{13}$ & 1.8074 & 1.6321 & 1.6321 & 1.0702 & 1.2756 & 1.5214 & 1.6654 & 1.0551 \\
\hline $\mathrm{IM}_{14}$ & 1.8114 & 1.1477 & 1.3475 & 1.2354 & 1.4435 & 1.3214 & 1.0766 & 1.0321 \\
\hline $\mathrm{IM}_{15}$ & 1.1214 & 1.7561 & 1.1832 & 1.7547 & 1.4123 & 1.2147 & 1.4471 & 1.1123 \\
\hline
\end{tabular}

TABLE 7: Haze gradient analyses of the proposed dehazing model.

\begin{tabular}{|c|c|c|c|c|c|c|c|c|}
\hline Img. & DCP & FVID & WT & TGV & CTT & $L_{1}$ & $\mathrm{CNN}$ & NSGA-BCP \\
\hline $\mathrm{IM}_{1}$ & 2.0496 & 2.2412 & 2.1259 & 1.8333 & 1.7798 & 2.0864 & 2.0224 & 1.7786 \\
\hline $\mathrm{IM}_{2}$ & 1.9477 & 1.9919 & 1.7394 & 2.2863 & 1.9414 & 1.9901 & 1.9109 & 1.7382 \\
\hline $\mathrm{IM}_{3}$ & 1.9081 & 2.0821 & 2.2017 & 1.8236 & 1.8699 & 2.0223 & 1.7489 & 1.7477 \\
\hline $\mathrm{IM}_{4}$ & 1.8541 & 1.9036 & 2.2311 & 1.8296 & 2.2209 & 2.0962 & 1.9231 & 1.8284 \\
\hline $\mathrm{IM}_{5}$ & 1.9743 & 1.8502 & 1.9164 & 2.1104 & 1.8947 & 2.0978 & 1.9491 & 1.8494 \\
\hline $\mathrm{IM}_{6}$ & 1.9285 & 2.2606 & 2.1083 & 2.0425 & 1.9375 & 2.2805 & 1.9785 & 1.9273 \\
\hline $\mathrm{IM}_{7}$ & 2.2466 & 2.2695 & 1.7853 & 1.7281 & 1.9208 & 1.9918 & 1.9867 & 1.7269 \\
\hline $\mathrm{IM}_{8}$ & 2.1197 & 2.1937 & 2.2231 & 2.1431 & 1.1752 & 1.9886 & 2.1691 & 1.7744 \\
\hline $\mathrm{IM}_{9}$ & 2.2834 & 1.8309 & 1.8698 & 1.8264 & 1.8384 & 2.1682 & 2.2709 & 1.8252 \\
\hline $\mathrm{IM}_{10}$ & 2.0828 & 1.8719 & 1.7288 & 1.7963 & 2.2443 & 2.0386 & 2.2368 & 1.7276 \\
\hline $\mathrm{IM}_{11}$ & 1.7864 & 1.8741 & 1.7926 & 2.1949 & 2.0954 & 1.8999 & 2.2163 & 1.7852 \\
\hline $\mathrm{IM}_{12}$ & 1.8189 & 2.2118 & 1.8735 & 2.1447 & 1.8708 & 1.8999 & 1.9918 & 1.8177 \\
\hline $\mathrm{IM}_{13}$ & 2.1464 & 2.1439 & 2.1122 & 2.1986 & 1.9486 & 2.2692 & 2.2327 & 1.9474 \\
\hline $\mathrm{IM}_{14}$ & 1.9264 & 2.0314 & 1.9543 & 1.8266 & 1.9892 & 1.8087 & 1.8531 & 1.8075 \\
\hline $\mathrm{IM}_{15}$ & 2.2915 & 1.9563 & 1.7242 & 2.0586 & 2.1525 & 2.2626 & 1.8594 & 1.7238 \\
\hline
\end{tabular}


TABLE 8: Peak signal-to-noise ratio (PSNR) analyses of the proposed dehazing model.

\begin{tabular}{lcccccccc}
\hline Img. & DCP & FVID & WT & TGV & CTT & $L_{1}$ & CNN & NSGA-BCP \\
\hline $\mathrm{IM}_{1}$ & 21.4352 & 21.3526 & 17.4847 & 25.2233 & 19.7988 & 25.9443 & 18.2123 & 27.1617 \\
$\mathrm{IM}_{2}$ & 19.3116 & 17.0435 & 20.2494 & 20.6626 & 23.4879 & 25.8382 & 17.2867 \\
$\mathrm{IM}_{3}$ & 18.8484 & 25.6161 & 23.9796 & 23.4317 & 23.2487 & 18.2346 & 22.2156 \\
$\mathrm{IM}_{4}$ & 18.2339 & 27.5254 & 26.7262 & 20.0566 & 24.6671 & 21.1583 & 16.8985 & 26.8378 \\
$\mathrm{IM}_{5}$ & 19.5562 & 22.7405 & 27.0682 & 26.7358 & 16.9676 & 17.7742 & 17.4003 & 28.7417 \\
$\mathrm{IM}_{6}$ & 24.6329 & 24.1535 & 24.2207 & 25.0819 & 18.6774 & 22.1306 & 19.8634 \\
$\mathrm{IM}_{7}$ & 19.8772 & 25.4108 & 25.7341 & 19.6746 & 24.0464 & 22.7067 & 21.4376 \\
$\mathrm{IM}_{8}$ & 23.7912 & 27.6807 & 21.8149 & 27.5865 & 21.3945 & 20.0567 & 25.7018 & 26.3036 \\
$\mathrm{IM}_{9}$ & 27.7838 & 26.8985 & 17.2131 & 21.0298 & 20.6644 & 20.2541 & 27.3327 & 28.9024 \\
$\mathrm{IM}_{10}$ & 19.3601 & 24.0618 & 27.6223 & 25.4101 & 21.8054 & 24.1566 & 26.9122 & 28.0055 \\
$\mathrm{IM}_{11}$ & 19.1564 & 24.7815 & 19.8693 & 23.0911 & 18.9015 & 21.2278 & 24.9664 & 26.1881 \\
$\mathrm{IM}_{12}$ & 25.6242 & 20.3746 & 22.8543 & 21.1459 & 21.6141 & 24.0739 & 19.1215 & 26.8459 \\
$\mathrm{IM}_{13}$ & 21.5967 & 18.4221 & 17.6245 & 27.7589 & 19.6873 & 24.5459 & 18.4737 & 28.9806 \\
$\mathrm{IM}_{14}$ & 25.2212 & 23.8978 & 21.1918 & 25.1502 & 23.8473 & 18.0318 & 17.5223 \\
$\mathrm{IM}_{15}$ & 26.5971 & 27.3211 & 27.0028 & 24.3435 & 18.3536 & 21.7065 & 20.2388 & 26.4429 \\
\hline
\end{tabular}

TABLE 9: Comparative analyses of the proposed dehazing model with metaheuristic techniques.

\begin{tabular}{llllllrr}
\hline Technique & \multicolumn{2}{c}{ CG } & \multicolumn{2}{c}{ NVE } & AG & \multicolumn{2}{c}{ PSNR } \\
\hline MOHO [40]-BCP & $2.42 \pm 0.077$ & $2.43 \pm 0.191$ & $2.19 \pm 0.111$ & $30.9 \pm 0.67$ & $0.883 \pm 0.079$ \\
MOEO [41]-BCP & $2.39 \pm 0.181$ & $2.24 \pm 0.189$ & $2.56 \pm 0.133$ & $31.3 \pm 1.23$ & $0.882 \pm 0.064$ \\
MOPSO [42]-BCP & $2.74 \pm 0.193$ & $2.16 \pm 0.115$ & $2.66 \pm 0.079$ & $29.9 \pm 1.18$ & $0.881 \pm 0.091$ \\
MOGWO [43]-BCP & $2.33 \pm 0.129$ & $2.42 \pm 0.137$ & $2.07 \pm 0.162$ & $30.2 \pm 0.77$ & $0.875 \pm 0.079$ \\
MOWO [44]-BCP & $2.38 \pm 0.061$ & $2.14 \pm 0.112$ & $2.38 \pm 0.101$ & $29.4 \pm 0.94$ & $0.873 \pm 0.098$ \\
NSGA-BCP & $2.51 \pm 0.172$ & $2.67 \pm 0.067$ & $2.69 \pm 0.136$ & $31.8 \pm 1.83$ & $0.887 \pm 0.082$ \\
\hline
\end{tabular}

TABLE 10: Structural similarity index metric (SSIM) analyses of the proposed dehazing model.

\begin{tabular}{lcccccccc}
\hline Img. & DCP & FVID & WT & TGV & CTT & $L_{1}$ & CNN & NSGA-BCP \\
\hline $\mathrm{IM}_{1}$ & 0.7502 & 0.7621 & 0.8674 & 0.7793 & 0.7619 & 0.8121 & 0.8067 & 0.8691 \\
$\mathrm{IM}_{2}$ & 0.8522 & 0.7959 & 0.8665 & 0.7255 & 0.8844 & 0.7894 & 0.8506 \\
$\mathrm{IM}_{3}$ & 0.8162 & 0.8257 & 0.7331 & 0.8518 & 0.7529 & 0.7478 & 0.8314 & 0.8861 \\
$\mathrm{IM}_{4}$ & 0.8715 & 0.7578 & 0.7952 & 0.7856 & 0.7384 & 0.8636 & 0.8344 & 0.8535 \\
$\mathrm{IM}_{5}$ & 0.8096 & 0.8764 & 0.8039 & 0.7401 & 0.8591 & 0.7449 & 0.7541 \\
$\mathrm{IM}_{6}$ & 0.7609 & 0.8439 & 0.8329 & 0.8803 & 0.7946 & 0.7783 & 0.8394 \\
$\mathrm{IM}_{7}$ & 0.8386 & 0.7307 & 0.8905 & 0.8617 & 0.7783 & 0.8983 & 0.7417 & 0.9081 \\
$\mathrm{IM}_{8}$ & 0.8365 & 0.8015 & 0.7511 & 0.8364 & 0.8228 & 0.8279 & 0.8141 & 0.8826 \\
$\mathrm{IM}_{9}$ & 0.7311 & 0.7582 & 0.7299 & 0.7662 & 0.7614 & 0.7832 & 0.8893 \\
$\mathrm{IM}_{10}$ & 0.7383 & 0.7258 & 0.7557 & 0.7393 & 0.7712 & 0.8029 & 0.7764 & 0.8982 \\
$\mathrm{IM}_{11}$ & 0.8988 & 0.7244 & 0.7391 & 0.8895 & 0.7345 & 0.8714 & 0.7234 \\
$\mathrm{IM}_{12}$ & 0.7908 & 0.8567 & 0.8973 & 0.8488 & 0.8235 & 0.7834 & 0.8265 & 0.8046 \\
$\mathrm{IM}_{13}$ & 0.7582 & 0.7423 & 0.8575 & 0.7468 & 0.8319 & 0.7338 & 0.4523 \\
$\mathrm{IM}_{14}$ & 0.7274 & 0.8645 & 0.8914 & 0.7522 & 0.7959 & 0.8781 & 0.7421 & 0.8905 \\
$\mathrm{IM}_{15}$ & 0.7277 & 0.8823 & 0.8005 & 0.8784 & 0.7616 & 0.7813 & 0.8444 & 0.8592 \\
\end{tabular}

(MOWO) [44]. We have applied the selected metaheuristic techniques on BCP to obtain the restored images. It has been observed that the proposed model achieves significantly better results as compared to the existing metaheuristic techniques.

\section{Conclusion}

A brightness channel prior (BCP) based dehazing model was implemented. The transmission map refinement was achieved using the gradient filter. The hyperparameters of BCP are tuned using NSGA. The obtained results revealed that BCP outperforms the competitive dehazing models in terms of contrast gain, new visible edges, average gradient, peak signal-to-noise ratio, and structural similarity index metric by $1.9379 \%, 1.3820 \%, 1.3289 \%, 1.9389 \%$, and $1.7392 \%$, respectively. Compared to the competitive models, BCP also minimizes the smog gradient, saturated pixels, and execution time by $1.8382 \%, 1.2372 \%$, and $0.8272 \%$, respectively. 


\section{Data Availability}

The data used to support the findings of this study are available from the corresponding author upon request.

\section{Ethical Approval}

The research was conducted according to the principles expressed in the Declaration of Hindawi.

\section{Conflicts of Interest}

The authors declare no conflicts of interest.

\section{References}

[1] M. Jian, Y. Yin, J. Dong, and W. Zhang, "Comprehensive assessment of non-uniform illumination for $3 \mathrm{~d}$ heightmap reconstruction in outdoor environments," Computers in Industry, vol. 99, pp. 110-118, 2018.

[2] S. Liu and Y. Zhang, "Detail-preserving underexposed image enhancement via optimal weighted multi-exposure fusion," IEEE Transactions on Consumer Electronics, vol. 65, no. 3, 2019.

[3] J.-P. Tarel and N. Hautiere, "Fast visibility restoration from a single color or gray level image," in Proceedings of 2009 IEEE 12th International Conference on Computer Vision, pp. 2201-2208, IEEE, Kyoto, Japan, September 2009.

[4] C. O. Ancuti, C. Ancuti, and P. Bekaert, "Enhancing by saliency-guided decolorization," in Proceedings of CVPR 2011, pp. 257-264, IEEE, Colorado Springs, CO, USA, June 2011.

[5] M. Jian, K.-M. Lam, and J. Dong, "Illumination-insensitive texture discrimination based on illumination compensation and enhancement," Information Sciences, vol. 269, pp. 60-72, 2014.

[6] Y. Zhang and S. Liu, "Non-uniform illumination video enhancement based on zone system and fusion," in Proceedings of 2018 24th International Conference on Pattern Recognition (ICPR), pp. 2711-2716, IEEE, Beijing, China, August 2018.

[7] X. Liu, H. Zhang, Y. Y. Tang, and J. X. Du, "Scene-adaptive single image dehazing via opening dark channel model," IET Image Processing, vol. 10, no. 11, pp. 877-884, 2016.

[8] I. Riaz, X. Fan, and H. Shin, "Single image dehazing with bright object handling," IET Computer Vision, vol. 10, no. 8, pp. 817-827, 2016.

[9] S. G. Narasimhan and S. K. Nayar, "Contrast restoration of weather degraded images," IEEE Transactions on Pattern Analysis and Machine Intelligence, vol. 25, no. 6, pp. 713-724, 2003.

[10] S. K. Nayar and S. G. Narasimhan, "Vision in bad weather," vol. 2, pp. 820-827, in 1999 Proceedings of the Seventh IEEE International Conference on Computer Vision, vol. 2, pp. 820-827, IEEE, Corfu, Greece, September 1999.

[11] B. Li, S. Wang, J. Zheng, and L. Zheng, "Single image haze removal using content-adaptive dark channel and post enhancement," IET Computer Vision, vol. 8, no. 2, pp. 131-140, 2014.

[12] D. Wang and J. Zhu, "Fast smoothing technique with edge preservation for single image dehazing," IET Computer Vision, vol. 9, no. 6, pp. 950-959, 2015.

[13] J. P. Oakley and B. L. Satherley, "Improving image quality in poor visibility conditions using a physical model for contrast degradation," IEEE Transactions on Image Processing, vol. 7, no. 2, pp. 167-179, 1998.
[14] D. Nair and P. Sankaran, "Color image dehazing using surround filter and dark channel prior," Journal of Visual Communication and Image Representation, vol. 50, pp. 9-15, 2018.

[15] K. He, J. Sun, and X. Tang, "Single image haze removal using dark channel prior," IEEE Transactions on Pattern Analysis and Machine Intelligence, vol. 33, no. 12, pp. 2341-2353, 2011.

[16] J. Bala and K. Lakhwani, "Single image desmogging using oblique gradient profile prior and variational minimization," Multidimensional Systems and Signal Processing, vol. 31, no. 9, pp. 1-17, 2020.

[17] M. Zhu, B. He, J. Liu, and J. Yu, "Boosting dark channel dehazing via weighted local constant assumption," Signal Processing, vol. 171, p. 107453, 2020.

[18] A. Mathias and D. Samiappan, "Underwater image restoration based on diffraction bounded optimization algorithm with dark channel prior,” Optik, vol. 192, p. 162925, 2019.

[19] D. Singh, V. Kumar, and M. Kaur, "Single image dehazing using gradient channel prior," Applied Intelligence, vol. 49, no. 12, pp. 4276-4293, 2019.

[20] Y. Jiang, C. Sun, Y. Zhao, and L. Yang, "Image dehazing using adaptive bi-channel priors on superpixels," Computer Vision and Image Understanding, vol. 165, pp. 17-32, 2017.

[21] Y. Wang, T.-Z. Huang, X.-L. Zhao, L.-J. Deng, and T.-Y. Ji, “A convex single image dehazing model via sparse dark channel prior," Applied Mathematics and Computation, vol. 375, p. $125085,2020$.

[22] G. Hou, J. Li, G. Wang, H. Yang, B. Huang, and Z. Pan, “A novel dark channel prior guided variational framework for underwater image restoration," Journal of Visual Communication and Image Representation, vol. 66, p. 102732, 2020.

[23] S. Ghosh, P. Shivakumara, P. Roy, U. Pal, and T. Lu, "Graphology based handwritten character analysis for human behaviour identification," CAAI Transactions on Intelligence Technology, vol. 5, no. 1, pp. 55-65, 2020.

[24] T. Wiens, "Engine speed reduction for hydraulic machinery using predictive algorithms," International Journal of Hydromechatronics, vol. 2, no. 1, pp. 16-31, 2019.

[25] J.-M. Guo, J.-Y. Syue, V. Radzicki, and H. Lee, “An efficient fusion-based defogging," IEEE Transactions on Image Processing, vol. 99, 2017.

[26] S. M. Yoon, "Visibility enhancement of fog-degraded image using adaptive total variation minimisation," The Imaging Science Journal, vol. 64, no. 2, pp. 82-86, 2016.

[27] B. Jiang, H. Meng, J. Zhao et al., "Single image fog and haze removal based on self-adaptive guided image filter and color channel information of sky region," Multimedia Tools and Applications, vol. 77, pp. 13513-13530, 2017.

[28] Z. Luan, Y. Shang, X. Zhou, Z. Shao, G. Guo, and X. Liu, "Fast single image dehazing based on a regression model," $\mathrm{Neu}$ rocomputing, vol. 245, pp. 10-22, 2017.

[29] X. Liu, H. Zhang, Y. ming Cheung, X. You, and Y. Y. Tang, "Efficient single image dehazing and denoising: an efficient multi-scale correlated wavelet approach," Computer Vision and Image Understanding, vol. 162, pp. 23-33, 2017.

[30] Y. Wu, Y. Qin, Z. Wang, X. Ma, and Z. Cao, "Densely pyramidal residual network for uav-based railway images dehazing," Neurocomputing, vol. 371, pp. 124-136, 2020.

[31] Q. Shu, C. Wu, Q. Zhong, and R. W. Liu, "Alternating minimization algorithm for hybrid regularized variational image dehazing," Optik, vol. 185, pp. 943-956, 2019.

[32] C. Hodges, M. Bennamoun, and H. Rahmani, "Single image dehazing using deep neural networks," Pattern Recognition Letters, vol. 128, pp. 70-77, 2019. 
[33] J. Zhang, X. Wang, C. Yang, J. Zhang, D. He, and H. Song, "Image dehazing based on dark channel prior and brightness enhancement for agricultural remote sensing images from consumer-grade cameras," Computers and Electronics in Agriculture, vol. 151, pp. 196-206, 2018.

[34] S. Emberton, L. Chittka, and A. Cavallaro, "Underwater image and video dehazing with pure haze region segmentation," Computer Vision and Image Understanding, vol. 168, pp. 145-156, 2018.

[35] F. Guo, X. Zhao, J. Tang, H. Peng, L. Liu, and B. Zou, "Single image dehazing based on fusion strategy," Neurocomputing, vol. 378, 2020.

[36] A. Alajarmeh, R. Salam, K. Abdulrahim, M. Marhusin, A. Zaidan, and B. Zaidan, "Real-time framework for image dehazing based on linear transmission and constant-time airlight estimation," Information Sciences, vol. 436-437, pp. 108-130, 2018.

[37] Y. Gao, Q. Li, and J. Li, "Single image dehazing via a dualfusion method," Image and Vision Computing, vol. 94, p. $103868,2020$.

[38] H. S. Basavegowda and G. Dagnew, "Deep learning approach for microarray cancer data classification," CAAI Transactions on Intelligence Technology, vol. 5, no. 1, pp. 22-33, 2020.

[39] R. Wang, H. Yu, G. Wang, G. Zhang, and W. Wang, "Study on the dynamic and static characteristics of gas static thrust bearing with micro-hole restrictors," International Journal of Hydromechatronics, vol. 2, no. 3, pp. 189-202, 2019.

[40] U. Yüzgeç and M. Kusoglu, "Multi-objective harris hawks optimizer for multiobjective optimization problems," BSEU Journal of Engineering Research and Technology, vol. 1, no. 1, pp. 31-41, 2020.

[41] M.-R. Chen and Y.-Z. Lu, "A novel elitist multiobjective optimization algorithm: multiobjective extremal optimization," European Journal of Operational Research, vol. 188, no. 3, pp. 637-651, 2008.

[42] P. K. Tripathi, S. Bandyopadhyay, and S. K. Pal, "Multiobjective particle swarm optimization with time variant inertia and acceleration coefficients," Information Sciences, vol. 177, no. 22, pp. 5033-5049, 2007.

[43] S. Khalilpourazari and S. H. R. Pasandideh, "Multi-objective optimization of multi-item eoq model with partial backordering and defective batches and stochastic constraints using mowca and mogwo," Operational Research, vol. 20, no. 2, pp. 1-33, 2018.

[44] I. R. Kumawat, S. J. Nanda, and R. K. Maddila, "Multiobjective whale optimization," in Proceedings of Tencon 2017-2017 IEEE Region 10 Conference, pp. 2747-2752, IEEE, Penang, Malaysia, November 2017.

[45] Y. Yuan, H. Xu, B. Wang, and X. Yao, “A new dominance relation-based evolutionary algorithm for many-objective optimization," IEEE Transactions on Evolutionary Computation, vol. 20, no. 1, pp. 16-37, 2015.

[46] K. Deb, A. Pratap, S. Agarwal, and T. Meyarivan, “A fast and elitist multiobjective genetic algorithm: Nsga-ii," IEEE Transactions on Evolutionary Computation, vol. 6, no. 2, pp. 182-197, 2002.

[47] M. Kaur, D. Singh, V. Kumar, and K. Sun, "Color image dehazing using gradient channel prior and guided 10 filter," Information Sciences, vol. 521, pp. 326-342, 2020.

[48] S. Osterland and J. Weber, "Analytical analysis of single-stage pressure relief valves," International Journal of Hydromechatronics, vol. 2, no. 1, pp. 32-53, 2019.

[49] B. Gupta, M. Tiwari, and S. S. Lamba, "Visibility improvement and mass segmentation of mammogram images using quantile separated histogram equalisation with local contrast enhancement," CAAI Transactions on Intelligence Technology, vol. 4, no. 2, pp. 73-79, 2019.

[50] A. Golts, D. Freedman, and M. Elad, "Unsupervised single image dehazing using dark channel prior loss," IEEE Transactions on Image Processing, vol. 29, pp. 2692-2701, 2020.

[51] C. O. Ancuti, C. Ancuti, C. De Vleeschouwer, and M. Sbetr, "Color channel transfer for image dehazing," IEEE Signal Processing Letters, vol. 26, no. 9, pp. 1413-1417, 2019.

[52] L. Li, Y. Dong, W. Ren et al., "Semi-supervised image dehazing," IEEE Transactions on Image Processing, vol. 29, pp. 2766-2779, 2020.

[53] H. Khan, M. Sharif, N. Bibi et al., "Localization of radiance transformation for image dehazing in wavelet domain," Neurocomputing, vol. 381, pp. 141-151, 2020.

[54] A. Galdran, J. Vazquez-Corral, D. Pardo, and M. Bertalmío, "Fusion-based variational image dehazing," IEEE Signal Processing Letters, vol. 24, no. 2, pp. 151-155, 2017.

[55] T. Cui, J. Tian, E. Wang, and Y. Tang, "Single image dehazing by latent region-segmentation based transmission estimation and weighted 11-norm regularisation," IET Image Processing, vol. 11, no. 2, pp. 145-154, 2017.

[56] Y. Gu, X. Yang, and Y. Gao, "A novel total generalized variation model for image dehazing," Journal of Mathematical Imaging and Vision, vol. 61, no. 9, pp. 1329-1341, 2019. 